\title{
Analytical challenges in characterization of high purity materials
}

\author{
K L RAMAKUMAR \\ Fuel Chemistry Division, Bhabha Atomic Research Centre, Mumbai 400 085, India
}

\begin{abstract}
Available analytical literature reveals that it is possible to identify a lot of procedures to carry out any determination using a plethora of analytical techniques. The fundamental analytical requirements for realizing the desired and acceptable information from a chemical analysis are representative nature of the sample, precision, accuracy, selectivity and sensitivity. These decide, to a larger extent, the selection of the most appropriate methodology in order to obtain chemical information from a system. A number of analytical methodologies are being used in the author's laboratory for carrying out trace elemental analysis as a part of chemical quality control. Quite a good number of analytical challenges with specific reference to the characterization of high purity materials of relevance to nuclear technology were addressed and methodologies were developed for trace elemental analysis of both metallic and non-metallic constituents. A brief review of these analytical challenges and the analytical methodologies developed and also the future needs of analytical chemist are presented in this paper.
\end{abstract}

Keywords. High purity materials; mass spectrometry; ion chromatography.

\section{Introduction}

The tremendous development of physical methods of analysis offers an impressive number of tools to simultaneously determine a large number of elements and compounds at very low concentration levels. Today's Analytical Chemistry provides necessary platform to address technical problems and to obtain correct information about chemical systems in order to take the most appropriate decisions for problem solving. The fundamental analytical requirements for realizing the desired and acceptable information from a chemical analysis are representative nature of the sample, precision, accuracy, selectivity and sensitivity. These decide, to a larger extent, the selection of the most appropriate methodology in order to obtain chemical information from a system. Though this, in principle, is a parameter of the soundness of the analytical capabilities, it can cause terrible drawbacks to those who try to apply a method to solve a specific problem without having a lot of time to evaluate all the advantages and problems inherent to the different methodologies. Despite their universal use, the instrumental analytical methods are usually relative methods. Reference materials are an absolute necessity. In recent years, the main efforts are focused on the development of new, more sophisticated methods of measurement based on physicochemical principles so as to bring down the limits of quantification almost to atomic level. It is also necessary to pay attention to other steps of the analytical process such as: (i) sampling and sample storage, (ii) sample pretreatment, (iii) selection of the most appropriate technique, (iv) hyphenation between analytical techniques,

klram@magnum.barc.ernet.in especially between separation and determination, and (v) data exploitation. This is all the more important and need to be addressed particularly when one is interested in determining ultra-trace amounts of elements at $10^{-12} \mathrm{~g}$ or less concentration levels. All the chemical or wet steps are too crucial to ignore at these levels. Furthermore, there is a definite need to improve upon the detection limits achievable and the lowest limits of quantification for material characterization required in the fields of nanotechnology, biosciences, and in fields such as art and archeology.

A number of analytical methodologies are being used in the author's laboratory for carrying out trace elemental analysis as a part of chemical quality control. These ranged from mass spectrometry (from the good old spark source mass spectrometry to quadrupole based inductively coupled plasma mass spectrometry), chromatography and other gas analysis systems. Most of these techniques have been and are being used routinely for chemical quality control measurements. Quite a good number of analytical challenges with specific reference to the characterization of high purity materials of relevance to nuclear technology were addressed and methodologies were developed for trace elemental analysis of both metallic and nonmetallic constituents. A brief review of these analytical challenges and the analytical methodologies developed and also the future needs of analytical chemist are presented in this paper.

\section{Analytical challenges in spark source mass spectrometry (SSMS)}

\subsection{Determination of hydrogen}

Despite some of its limitations, SSMS with conventional 
photo plate detection system can still be considered as an attractive solid sample analysis technique. With MattauchHerzog ion optics geometry, simultaneous analysis of almost all elements across the periodic table has become possible with reasonably low limits of detection. However, hydrogen cannot be determined because of its predispersion in the analyser itself. This robs away the multi-elemental analysis capability of SSMS. Hydrogen is one of the important specification elements in zircaloy, an indispensable material in nuclear technology. Its simultaneous determination along with other trace elements is certainly an attractive proposition. A two-level exposure technique was developed to determine hydrogen also by SSMS (Ramakumar et al 1986).

In the two-level exposure technique, the photo plate is exposed at two different magnetic field settings. One longitudinal half is exposed at a setting of $1.5 \mathrm{~T}$ to cover the $\mathrm{m} / \mathrm{z}$ values from 6 to 240 and the other half is exposed at $0.4 \mathrm{~T}$ to cover the mass range from $\mathrm{m} / \mathrm{z} 1$ to 30 . Hydrogen is determined with respect to an internal reference material (IRM) at lower magnetic field setting. This IRM is in turn determined with respect to the matrix elements $\mathrm{Zr}$ at higher field setting. Concentration of hydrogen is simply then given by $[\mathrm{H}]_{\mathrm{Zr}}=[\mathrm{H}]_{\mathrm{IRM}} \times[\mathrm{IRM}]_{\mathrm{Zr}}$.

Either nitrogen, carbon or even a multiply charged $\mathrm{Zr}^{+n}$ mass peak can be chosen as internal reference material and the results for hydrogen were shown to be consistent and also agreed very well with those obtained by another independent technique based on inert gas fusion. This two-level exposure technique was successfully employed for the analysis of a failed zircaloy clad sample to ascertain the source of hydrogen.

It should be mentioned that due to larger dispersion at lower magnetic field setting, it was possible to resolve the peaks at $\mathrm{m} / \mathrm{z} 28$ due to $\mathrm{Ni}_{2}^{+}, \mathrm{Si}^{+}$, and ${ }^{56} \mathrm{Fe}^{+2}$ so that $\mathrm{Si}$ could be determined without any difficulty.

\subsection{Determination of phosphorus in zircaloy and $\mathrm{Zr}-\mathrm{Nb}$}

Normally whenever there are isobaric interferences at a particular $\mathrm{m} / \mathrm{z}$, an interference-free $\mathrm{m} / \mathrm{z}$ is selected. This is not always possible as in the case of phosphorus, which is monoisotopic. Multiply charged $\mathrm{Nb}$ ions $\left({ }^{93} \mathrm{Nb}^{+3}\right)$ and $\mathrm{Ni}\left({ }^{62} \mathrm{Ni}^{+2}\right)$ ions cause interference at $\mathrm{m} / \mathrm{z} 31$ corresponding to ${ }^{31} \mathrm{P}^{+}$. The resolution achievable in SSMS was not sufficient to resolve these from ${ }^{31} \mathrm{P}^{+}$mass peak. With a view to assessing the extent of interference from $\mathrm{Nb}^{+3}$, charge distribution investigations in the case of $\mathrm{Nb}^{+n}$ were carried out to establish correlations and the intensity at $\mathrm{m} / \mathrm{z} 31$ could be corrected for $\mathrm{Nb}^{+3}$ interference (Sant et al 1996). This method can be extended to determine other monoisotopic elements also.

\section{Quadrupole mass spectrometry for analysis of gases}

\subsection{Determination of hydrogen/deuterium in zircaloy}

Zircaloy and $\mathrm{Zr}-2.5 \% \mathrm{Nb}$ are being used as coolant channels in pressurized heavy water reactors (PHWRs). The life expectancy of these coolant channels critically depends on the extent of deuterium pick-up by them during reactor operation. The ageing management, therefore, requires that this deuterium pick-up rate should be quantified for the preventive measures to be taken for the smooth operation of the reactor. As hydrogen/deuterium has very limited solubility in zircaloy $(\sim 1 \mathrm{ppm}$ at room temperature), excess would be precipitated as a separate phase leading to hydrogen embrittlement resulting in reduced lifespan for these coolant channels. The whole exercise is to analyse hydrogen/deuterium pick up in wafer thin samples sliced from the interior of the coolant channels so that the integrity can be maintained for their continuous usage. Conventional technique such as IGF can only give total hydrogen or hydrogen equivalent (HEq) but not the deuterium pick-up separately. Moreover, in these dedicated instruments with TCD as the detection system, even this HEq is prone to errors as the thermal conductivities of hydrogen and deuterium differ by almost $30 \%$ (Ramakumar et al 2004). Further, analysis on small sample sizes lead to larger uncertainties. Other technique such as differential scanning calorimetry (DSC) also gives only total hydrogen equivalent. Mass spectrometry is the only technique that can give isotopic information. Here the challenge has been getting isotopic information on sliver samples weighing about only $10-20 \mathrm{mg}$ with reasonable precision and accuracy (5\% or less). A hot vacuum extraction set up has been designed, fabricated and commissioned and coupled to a quadrupole mass spectrometer (HVE-QMS) for this purpose (Sesha Sayi et al 1998). As low as $0.4 \mathrm{ppm}$ of deuterium pick up could be measured using this apparatus.

\subsection{Internal isotope dilution mass spectrometry (IIDMS) for deuterium determination}

An interesting offshoot of the HVE-QMS technique has been the development of IIDMS methodology for deuterium determination. Instead of using an external deuterium spike, in this method, deuterium spike is internally generated (Sayi et al 2002a) from a portion of the evolved gases from HVE-QMS. The developed method is specific for hydrogen/deuterium determination only but obviates the need for an external spike.

\subsection{Determination of sulphur in $\mathrm{ThO}_{2}$}

Sulphur has been identified as an important specification element as excess of the same would lead to cracking of $\mathrm{ThO}_{2}$ pellets during sintering due to formation of $\mathrm{H}_{2} \mathrm{~S}$. As $\mathrm{ThO}_{2}$ is refractory, its quantitative dissolution, though not impossible but very difficult, has to be ensured for the determination of sulphur. Direct solid analysis is desirable in this case. A technique known as combustion 
extraction-quadrupole mass spectrometry has been developed for the determination of sulphur in $\mathrm{ThO}_{2}$ samples (Sayi et al 2002b). The vacuum extraction system designed and fabricated earlier has been suitably modified to incorporate the combustion step. $\mathrm{ThO}_{2}$ sample is mixed with a known amount of an oxidizing material (e.g. nuclear pure $\mathrm{UO}_{3}$ ) and is heated at $\sim 1300 \mathrm{~K}$. The evolved gases are collected in a known volume and the gas composition is determined by quadrupole mass analyser. The concentration of sulphur is arrived at using gas laws from the amount of $\mathrm{SO}_{2}$ formed. Very low concentrations of sulphur (sub ppm) could be determined by this method.

\section{Halogens by ion chromatography}

\subsection{Fluoride determination in $(U, P u) C$}

Pyrohydrolysis of the sample followed by ion chromatography is the preferred method in the author's laboratory for the determination of $\mathrm{F}^{-}$, and $\mathrm{Cl}^{-}$in uranium and plutonium oxides. The method is straightforward and very low detection limits of $<5 \mathrm{ppb}$ could be achieved. But in the case of carbide samples, problems were encountered during the pyrohydrolysis stage. Fluoride values were higher than expected. Ion chromatograms showed very sharp peaks at fluoride elution indicating no possible coelution of other anions if any. But detailed investigations on the pyrohydrolysis of carbide samples showed that in addition to the halogen acids, they also produced many aliphatic acids, prominent among them being acetic and oxalic acids. It was found that acetate has exactly the same retention time as that of fluoride resulting in interference. Change of mobile phase to $\mathrm{NaOH}$ eliminated this interference (Jeyakumar and Ramakumar 2002).

\section{Inductively coupled plasma mass spectrometry (ICPMS)}

\subsection{Determination of iron}

The advent of ICP-MS has been one of the most fortuitous occurrences in the field of analytical chemistry. Many variants of this technique are now commercially available at the disposal of the analytical chemist and the technique has matured into a widely accepted method for ultra-trace multielemental analysis. Even though its multielemental analysis capabilities are undisputed, there are still many irritants e.g. in the form of molecular ion interferences due to nebuliser gas Ar. Most significant among these are the interference of $\mathrm{ArO}^{+}$at ${ }^{56} \mathrm{Fe}^{+}$mass peak. Cold plasma conditions and the shielded plasma torch have been resorted (Stuart Georgitis et al 1996) to eliminate this interference. In our laboratory, we studied the formation and stability of this background signal due to
$\mathrm{ArO}^{+}$ion. It is seen that after about $60 \mathrm{~min}$ into the analysis sequence, the signal became stable and reproducible (Deb et al 2003). When iron peak was monitored after this period, the results were found to be reproducible and the blank correction could be carried out accurately.

\section{Today's technological and analytical requirements}

In recent years, the main efforts are focused on the development of new, more sophisticated methods of measurement based on physicochemical principles so as to bring down the limits of quantification almost to atomic level. Now-a-days, the need is to look for the following capabilities in an analytical technique: (a) low detection limits (single atom?), (b) excellent precision $(<0.1 \% \mathrm{rsd})$ at even $<0.1 \%$ level, (c) broad dynamic range $\left(>10^{7}\right)$, (d) no spectral or matrix interferences, (e) complete, simultaneous elemental coverage, (f) isotope-analysis capability, (g) absolute (standardless) analysis, (h) no sample preparation, (i) macro, micro, or transient samples amenable to speciation, (j) spatial resolution in solid samples, (k) rapid (10 s per sample), (1) inexpensive (initial and continuing), (m) simple, automated, and compact, and (n) sample state-independence (solid, liquid or gas). The requirements may seem to be too elaborate but should in no time become absolutely mandatory in the fields of nanotechnology or in biosciences. When we are looking for the determination of a few thousands of atoms/species/ analyte (fg to ag levels), it is meaningless to report values as $<1 \mathrm{pg}$. Rigorous statistics is required at this level to assign true or expected value.

\subsection{Limitations of currently available ICP-MS systems}

Despite being a very good analytical technique, ICP-MS exhibits shortcomings. In particular, although detection limits are low in terms of concentration, they are often inadequate on an absolute (mass) basis for extremely tiny or precious samples. Moreover, it does not provide adequate precision for isotope ratio measurements if many isotopes are to be determined. Isobaric overlaps (spectral interferences) can also be very troublesome in some situations. Similarly, matrix interferences can adversely affect many determinations. Further, quadrupole mass filters and sector-field mass spectrometers of the sort commonly employed for ICP-MS are inherently sequential instruments, which limits their speed, precision, and applicability to many kinds of samples or sampleintroduction equipment. Magnetic sector multicollection detection systems with as many as 9 faraday cups are now commercially available with ICP system mitigating some of these limitations (Walder et al 1993; White et al 2000). It is in the area of high-speed transient measurements that ICP-MS perhaps suffers its greatest weakness. 
Lack of absolute (standardless) analysis, too, is a shortcoming, especially in the analysis of solid samples such as often performed with laser ablation. In turn, these limitations complicate sample preparation and make it difficult for ICP-MS to be employed with micro or transient samples like those produced by flow-injection, electrothermal vapourization, laser ablation, or chromatography. This latter difficulty makes multielemental speciation unnecessarily inconvenient. When these sampling devices are employed, the user must choose between broad elemental or isotopic coverage and signal-to-noise ratio $(\mathrm{S} / \mathrm{N})$. In turn, compromised $\mathrm{S} / \mathrm{N}$ means lower precision or poorer detection limits. Many of these remaining complications can be overcome by use of simultaneous detection of analyte species. Simultaneous detection inherently provides a number of important benefits. Because all the channels are being monitored all the time, precision and detection limits improve as the square root of the number of channels that are being monitored. As there are about 207 isotopes that are normally monitored, if one were to monitor every one of those isotopes, simultaneous coverage will therefore improve precision and detection limits by roughly a factor of 14 for a fixed observation time. In addition, simultaneous detection can result in improved precision when ratioing techniques are utilized (Vanhaecke et al 1999). This advantage arises from the fact that much of the noise that is encountered in ICP-MS is correlated; that is, it affects all elemental signals in much the same way. Thus, the signal fluctuations from one element tend to follow those of others closely in time; ratioing the signals in perfect time registry can then compensate for the fluctuations, whereas any temporal offset (such as that produced by sequential scanning) cannot fully compensate. Similarly, simultaneous detection avoids a problem commonly termed "spectral skew". Spectral skew arises when a transient sample is being examined. In such a transient, the sample concentration changes continuously, so mass-spectral peaks measured at one point in the transient correspond to a different instantaneous sample concentration than those examined at any other point in time. The changing concentration therefore leads to quantitation errors that are difficult to correct. Lastly, higher sample throughput and improved background detection and correction naturally flow from simultaneous detection.

From this brief analysis, it seems that the most critical challenges in ICP-MS are the following: borderline precision for isotope-ratio measurements, especially in a multielement mode; the existence of troublesome matrix and spectral interferences; difficulty with micro and transient samples; consequently, compromised speciation; the need for matrix-matched standards when laser ablation is employed; and speed that is limited by a sequentialscanning process. Of course, every one of these challenges represents an opportunity-for research, discovery, and improvement.

\subsection{Innovations in available analytical instrumentation}

Efforts are being made to make new instrumentation available to analytical chemists to address many of the envisaged challenges. For example, an ICP system coupled to a time-of-flight mass spectrometer, provides excellent detection limits, resolving power (about 1200), better than commercial quadrupole mass filters, precision of atleast $0.02 \%$ rsd in a ratioing mode, and extraordinarily high speed for use with transient sampling devices such as flow injection generating between 20,000 and 30,000 complete elemental mass spectra per second (Sturgeon et al 2000). The second instrument is based on a sector-field mass spectrometer but, unlike other such units, is equipped with a focal-plane array detector. So equipped, the system can detect a broad mass range at once.

6.2a Flow injection-ICP-TOFMS: It has long been realized that flow injection is a natural complement to ICP-MS for elemental analysis (Dean et al 1988). However, the high speed and lack of spectral skew available from a TOFMS enhances this capability. Not only does ICP-TOFMS enable the entire elemental and isotopic pattern of a sample to be followed during the course of an FIA transient, it also permits high-precision isotope ratios to be measured at the same time and allows the true shape of an FI peak to be traced.

The approach (Hieftje et al 2001) to interference-free analysis in flow injection mode relies upon two facts: (i) ideally, the time-dependent change in concentration for all elements should be the same in flow injection; and (ii) matrix interferences in ICP-MS depend upon the absolute concentration of the interfering species and not on the interferent/analyte concentration ratio. Because the concentration of all analyte species changes proportionately during FI, and because TOFMS enables all the concentrations to be sampled at exactly the same time, the ratio of any two analyte signals should be constant at all times during the FI pulse, in the absence of an interference. In the presence of interfering species, its concentration, too, will change in direct proportion to the concentrations of the analyte species. Because the level of interference depends upon the absolute concentration of the interfering species, its presence will be apparent and therefore troublesome only at the FI peak. Earlier or later during the FI pulse, the interference should vanish for all practical purposes. Because analyte elements differ in their susceptibility to each interference, the analyte ratios should therefore exhibit a deviation from a constant value during the peak of the FI transient, which later returns to the same constant value they display during the initial, low-concentration portion of the transient. Because the time-dependent concentration of all species during the pulse is known, this return to a constant level indicates the dilution of the original sample that is necessary to overcome the interference. The signal of each analyte 
species can also be measured directly at the time the signal ratios return to a constant level. Again, because the concentration vs time profile of the FI process is known, the original analyte concentration can be determined as easily from this point as from the peak of the FI pulse. In this way, interference-free measurements should be possible from even a single sample injection.

6.2b Laser ablation-ICPMS: A standardless approach to quantitative analysis: In laser ablation ICPMS (LAICPMS), twin advantages of time resolution capability and getting a complete elemental mass spectrum for each input ion packet are exploited. These features obviate the necessity of matrix-matched solid standards in laserablation (LA) ICP-TOFMS. Ordinarily, quantitation in LA-ICP-TOFMS is compromised by changes in laser power, sample surface albedo, and other factors that alter the interaction between the laser beam and the sample. Although the best method to overcome these difficulties has been through use of matrix-matched standards (Van Heuzen and Morsink 1991), the lack of availability of such standards has caused users to turn to alternative techniques. These techniques include normalization based on measurements of the ablated aerosol (Baker et al 1998) or of the acoustic pulse (Pang et al 1991) generated by the action of the laser on the sample surface. This limitation can be overcome by recognizing that the sum of the peaks in a complete elemental mass spectrum should be proportional to the mass of sample that has been ablated. Normalization of each mass spectral peak by this summed spectrum then not only provides an excellent means of compensating for pulse-to-pulse variations in the laser, but also permits virtually standardless analysis to be performed. In essence, the ratio of a particular isotopic peak to the sum of all such peaks in the mass spectrum is directly the concentration of that isotope within the original sample. Of course, for this procedure to be effective, it is necessary to take account of slight variations in ionization efficiency from element to element and also the natural mass bias that occurs in any mass spectrometer.

One approach has been to employ a number of different alloys for standardization and to measure a range of elements in each (Hieftje et al 2001). A mass-bias factor and an empirical sensitivity value are then extracted from these data and applied to subsequent samples. It has been shown that a single set of such sensitivity and mass-bias factors allows the same calibration curve to be used with high fidelity for a broad range of sample types. Hieftje et al (2001) have adopted this approach and shown that for a principal matrix component aluminum, cobalt, copper, iron, nickel, and brass, despite a factor-of-six difference in the amount of material ablated from these different types of matrix, samples can be analysed with a single laser pulse that consumes as little as $13 \mathrm{ng}$ and with a depth resolution of the order of $200 \mathrm{~nm}$. Applied to stan- dards not in the original calibration set, the method offers semi-quantitative accuracy to within $3 \%$ for elements present at high concentration (above 10\%) and less than $10 \%$ error for elements that range between $0 \cdot 1$ and $10 \%$ in composition (with the exception of $\mathrm{Zn}$, which undergoes substantial fractionation). Current limitations of the method include a restriction to metal-alloy samples, because of the greater difficulty of measuring non-metals by ICP-MS. Limited dynamic range (three orders of magnitude) is another drawback of this technique at present.

6.2c Array detector atomic mass spectrometer (ADAMS): Most atomic emission spectrometers that are now being sold employ focal-plane array detectors. In order to avoid serious spectral interferences, atomic spectra must be measured at resolution levels of the order of $1 \mathrm{pm}$ and over a spectral range of roughly $600 \mathrm{~nm}$. Atleast 6,00,000 resolution elements are therefore needed to cover the full atomic emission range at the desired resolution. It is attractive to consider using a linear detector array to acquire a full atomic mass spectrum. In quadrupole mass filters, a unit-mass resolution is adequate for most applications. Further, there are only about 207 isotopes that would commonly be measured in atomic mass spectrometry, even if coverage across the entire periodic table were needed. If as many as five points across each massspectral peak were then desired, a simple 1-inch $(2.5 \mathrm{~cm})$ 1024-element linear array detector should suffice. At present, such systems are available at extremely low cost. But that would result in a resolution of about $0.2 \mathrm{amu}$ only, which may not be adequate to eliminate isobaric interferences in majority of cases.

If on the other hand, the spatially dispersive mass spectrometers that are now available are considered, they produce a mass spectrum that varies quadratically rather than linearly with mass-to-charge ratio. As a result, mass-spectral peaks are crowded together at one end of the mass scale but separated by a great distance at the other. As a result, if adequate mass resolution is to be achieved at the cluttered end of the spectrum, a great deal of wasted detector space would result at the other.

Hieftje et al (2001) circumvented this problem by dividing the atomic mass range into two segments, each of which has a ratio of upper to lower mass that is roughly the same. They have designed an instrument that can operate alternately over the mass range from $7 \mathrm{Li}$ to $39 \mathrm{~K}$ (ratio of $39 / 7=5 \cdot 6$ ) and from $41 \mathrm{~K}$ to $238 \mathrm{U}$ (ratio of $238 / 41=5 \cdot 8$ ). Conveniently, the two ranges avoid the troublesome peak at $40 \mathrm{u}$, ordinarily saturated because of the strong $\mathrm{Ar}^{+}$signal in ICP-MS. Switching between the two ranges is accomplished in a straightforward fashion by adjusting the accelerating voltage in the spectrometer. These authors designed an extremely compact instrument ( $80 \mathrm{~cm}$ in length) with Mattauch-Herzog ion optics geometry but offering outstanding performance. Its resolving power is atleast as good as would be expected 
from a quadrupole mass filter but, because it employs a higher accelerating voltage, it generates higher ion currents and low detector noise. Typical sensitivities with an ICP source are $>10^{8} \mathrm{cps} / \mathrm{ppm}$-isotope, and when an electron multiplier detector is used (in scanning mode only), detection limits are generally in the tens of parts per quadrillion range $\left(10^{-15}\right)$.

Now-a-days, a new generation of multichannel array detectors with extremely low background noise, for mass spectrometry is on the anvil. The detectors will rely upon Faraday cups for ion collection. This allows the detector to interrogate each channel as ions are being collected and to accumulate ions until a desired signal-to-noise ratio is achieved. Because some ions will naturally be arriving at a far greater rate than others, owing to their higher concentration in a sample, their channels can be read more frequently and thus avoiding their saturation. At the same time, weaker channels can be allowed to accumulate signal steadily. Thus, it becomes possible to extend the dynamic range of the instrument almost without limit realizing extremely low detection limits of the order of $10^{-15}$ or less.

It is anticipated that the detectors that will eventually be commercially available will be of a relatively small size, so they can fit not only along the flat focal plane of a Mattauch-Herzog spectrometer, but also they will be able to fit around the curved focal region of other massspectrometer designs. Further, because each detector chip will then be individual, it can be read extremely rapidly, at rates atleast as high as 1000 spectra per second. In many ways, this technology will offer the same benefits as the high-speed ICP-TOFMS systems.

\section{Laser induced breakdown spectrometry (LIBS)}

Laser spectroscopic techniques are very versatile for chemical analysis because they offer real-time monitoring capabilities with high analytical sensitivity and selectivity (Sjostrom and Mauchien 1991). These techniques are valuable for laboratory research. However, to take an instrument or technology into the field requires another level of research and development to ensure ruggedness, stability, reliability, a small instrument footprint, and calibration algorithms that have been tested for a variety of matrices and that is the ultimate goal. The ability of LIBS to provide rapid multielemental microanalysis of bulk samples (solid, liquid, gas, aerosol) in the parts-permillion (ppm) range with little or no sample preparation has been widely demonstrated (Martin and Cheng 2000). The instrumentation and operation of a LIBS system is simpler than some of the more sensitive techniques, and analysis times on the order of minutes for LIBS make it more amenable for real-time analyses of chemical processes. Although calibration standards are required for quantitative analysis, the generation of a single calibra- tion curve will suffice for analysis of samples in a similar matrix. The main attributes of this technique are: (a) solids, liquids and gases can be analysed without sample preparation, (b) in situ, real-time (rapid), noninvasive analysis (only a small amount of material is used in the analysis (ng to mg), almost an NDT method), (c) stand-off analysis (only optical access is required), (d) field-portable and (e) high resolution $(\mathrm{mm})$.

The method is simple. All that is required is a laser, a lens for focusing the laser light onto the sample, a lens or fibre optic for the collection of the emitted light, a spectrograph to separate the light by wavelength, an image intensifier, and a photodiode detector. The output can then be displayed or used as input to an automated system. Time-gated detectors are employed which allow the optical emission from the laser plasma to be recorded at some time delay after the laser pulse. This is important since the characteristic atomic and ionic emission lines only start to appear after the plasma has expanded and cooled. Because LIBS is essentially an all-optical technique, only optical access to the material is required to carry out an analysis. These features make LIBS a particularly attractive solution for analysis of hazardous or high-temperature materials, or for deployment in hostile environments. At present, detection limits are not comparable to those obtained in the conventional techniques. But the method holds tremendous potential.

\section{Conclusions}

Chemical analysis is a critically important enabling technology essential to every phase of chemical science, product and process development, and manufacturing control. The conduct of analytical chemistry has been transformed by advances in high-field superconducting magnets, multiplewavelength lasers, multiplex array detectors, atomic-force and scanning-tunneling microscopes, non-scanning spectral analysis (for example, Fourier transform), and the integration of computers with instrumentation (American Chemical Society 1996). At the same time new knowledge and insights about existing and new systems, developed as a result of advances in chemical measurement over the past two decades, have greatly accelerated progress in chemical science, biotechnology, materials science, and process engineering. Impressive achievements have been made in the resolution, sensitivity, and specificity of chemical analysis. Further, the inclusion of analytical specialists as full partners greatly enhances the efficiency of research teams in chemical synthesis, surface science, catalysis, nanostructure science and technology, and environmental chemistry. While significant progress has been made in moving process analytical measurements from the laboratory to the manufacturing line, more real-world chemical measurements are still conducted off-line. However, the isolation of sophisticated methods of chemical analysis from the environments in 
which they are most needed-ranging from R\&D laboratories through manufacturing facilities - is a major limitation in present-day chemical measurements.

\section{References}

American Chemical Society 1996 Technology Vision 2020

Baker S A, Smith B W and Winefordner J D 1998 Appl. Spectrosc. 52154

Dean J R, Ebdon L, Crews H M and Massey R C 1988 J. Anal. At. Spectrom. 3349

Deb S B, Raman V A, Saxena M K and Ramakumar K L 2003 Silver jubilee national symp. mass spectrom, Goa (Mumbai: Indian Society for Mass Spectrometry)

Hieftje G M et al 2001 Pure Appl. Chem. 731579

Jeyakumar S and Ramakumar K L 2002 Int. conf. characterization and quality control of nuclear fuels $(C Q C N F-2002)$ (Hyderabad: NFC, IAEA)

Martin M Z and Cheng M D 2000 Appl. Spectrosc. 541279

Pang H M, Wiederin D R, Houk R S and Yeung E S 1991 Anal. Chem. $\mathbf{6 3} 390$

Ramakumar K L, Raman V A, Sant V L, Shah P M, Kavimandan V D and Jain H C 1986 Anal. Lett. 192147

Ramakumar K L, Sesha Sayi Y, Shankaran P S, Chhapru G C, Yadav C S and Venugopal V 2004 Indian J. Chem. Technol. 11865
Sant V L, Raman V A, Ramakumar K L, Kavimandan V D, Aggarwal S K and Jain H C 1996 Proc. seventh nat. symp. mass spectrom. (7th NSMS) (Gwalior: DRDE) p. 465

Sesha Sayi Y, Ramakumar K L, Rajendra Prasad, Yadav C S, Shankaran P S, Chhapru G C and Jain H C 1998 J. Radioanal. Nucl. Chem. Articles 2385

Sayi Y S, Yadav C S, Shankaran P S, Chhapru G C, Ramakumar K L and Venugopal V 2002a Proc. symp. zirconium-2002 (Mumbai: BARC) p. 471

Sayi Y S, Shankaran P S, Yadav C S, Chhapru G C, Ramakumar K L and Venugopal V 2002b Proc. Nat. Acad. Sci. (India) 72 241

Sjostrom S and Mauchien P 1991 Spectra Chimica Acta B15 153

Stuart Georgitis, Stephen Anderson and Michael Plantz 1996 Tran T. Nham, Varian Application Notes 007

Sturgeon R E, Lam J W H and Saint A 2000 J. Anal. At. Spectrom. 15607

Vanhaecke F, Moens L, Dams R, Allen L and Georgitis S 1999 Anal. Chem. 713297

Van Heuzen A A and Morsink J B W 1991 Spectrochim. Acta B46 1819

Walder A J, Platzner I and Freeman P A 1993 J. Anal. At. Spectrom. 819

White W M, Albarede F and Telouk P 2000 P. Chem. Geol. 167 257 